\title{
Acute lower limb ischemia due to thrombo-embolic arterial occlusions in two previously healthy men with markedly elevated $L p(a)$
}

\author{
Gabriella V Bedarida, Ulrich Hoffmann and Federico Tatò
}

\begin{abstract}
Lipoprotein (a) $(\mathrm{Lp}(\mathrm{a}))$ is a well-documented risk factor for atherosclerotic cardiovascular disease. Its role in acute thrombo-embolic occlusions of peripheral arteries is not known. We describe two cases of multiple, acute, peripheral arterial occlusions in two previously healthy men with markedly elevated Lp(a). Both cases had unsatisfactory results after percutaneous and surgical revascularization procedures. Experience yielded in these two cases suggests that when an unfavorable outcome occurs in a peripheral artery disease patient in the absence of the regular risk factors, $L p(a)$ should be determined and its role investigated.
\end{abstract}

Key words: acute limb ischemia; arterial thrombosis; Lp(a); myointimal hyperplasia; peripheral artery disease

\section{Introduction}

Elevated lipoprotein (a) (Lp(a)) is an established risk factor for atherosclerosis and has been linked to premature coronary artery disease as well as stroke and peripheral artery disease (PAD). ${ }^{1-3} \mathrm{Lp}$ (a) possesses unique antifibrinolytic and prothombotic properties, and may play an important role in vascular thrombosis. ${ }^{1,4}$ However, elevated Lp(a) has not previously been recognized as a risk factor for acute thrombo-embolic occlusions of peripheral arteries. We report the cases of two adult men with markedly elevated Lp(a) and multifocal thrombo-embolic occlusions of the iliaco-femoral and popliteo-crural arteries in the absence of advanced atherosclerosis.

\section{Case reports}

\section{Case 1}

A 54-year-old previously healthy white male presented to our clinic with a 3-week history of right-sided calf

\footnotetext{
Division of Vascular Medicine, Ludwig-Maximilians University Hospital, Munich, Germany

Address for correspondence: Ulrich Hoffmann, Division of Vascular Medicine, Medizinische Poliklinik, Ludwig-Maximilians University, Pettenkoferstrasse 8a, D-80336 Munich, Germany. Tel: +49 895160 3590; Fax: +49 895160 4494; E-mail: ulrich. hoffmann@med.uni-muenchen.de
}

and foot claudication. His past medical history was significant only for a recently developed impotence, for which he had received three monthly testosterone injections. He had no history of smoking, diabetes, hypertension, hypercholesterolemia, and no family history of premature cardiovascular disease.

The physical examination and non-invasive vascular laboratory tests showed bilateral, distal PAD with impaired hemodynamic function at the level of the right calf and left foot. Duplex ultrasound showed hypoechogenic occlusion of the right popliteal artery in the absence of arteriosclerosis or aneurysm. The patient underwent an extensive work-up including a 24-hour Holter monitor, transesophageal echocardiogram and aortic imaging to rule out embolic disease. Laboratory tests including markers of inflammation (ESR, CRP), electrophoresis, antinuclear and anticardiolipin antibodies and tests for thrombophilia (fibrinogen, ATIII, protein $\mathrm{C}$ and $\mathrm{S}$, activated protein $\mathrm{C}$ ) were normal. Homocysteine was $13.2 \mu \mathrm{mol} / \mathrm{l}$ (normal values $<13.9 \mu \mathrm{mol} / \mathrm{l}$ ). Lipid and lipoprotein analysis showed normolipidemia (total cholesterol $203 \mathrm{mg} / \mathrm{dl}$, LDL-cholesterol $120 \mathrm{mg} / \mathrm{dl}$, HDL-cholesterol $55 \mathrm{mg} / \mathrm{dl}$, triglycerides $153 \mathrm{mg} / \mathrm{dl})$. Lp(a) was measured with a commercial turbidometric immunoassay (Wako, Japan) with a normal reference range of $<30 \mathrm{mg} / \mathrm{dl}$ and it was markedly elevated at $86 \mathrm{mg} / \mathrm{dl}$.

Digital subtraction angiography (DSA) showed occlusion of the popliteal artery of the right leg (Figure 1), and occlusion of the posterior and anterior tibial arteries of the left leg with thrombo-embolic 


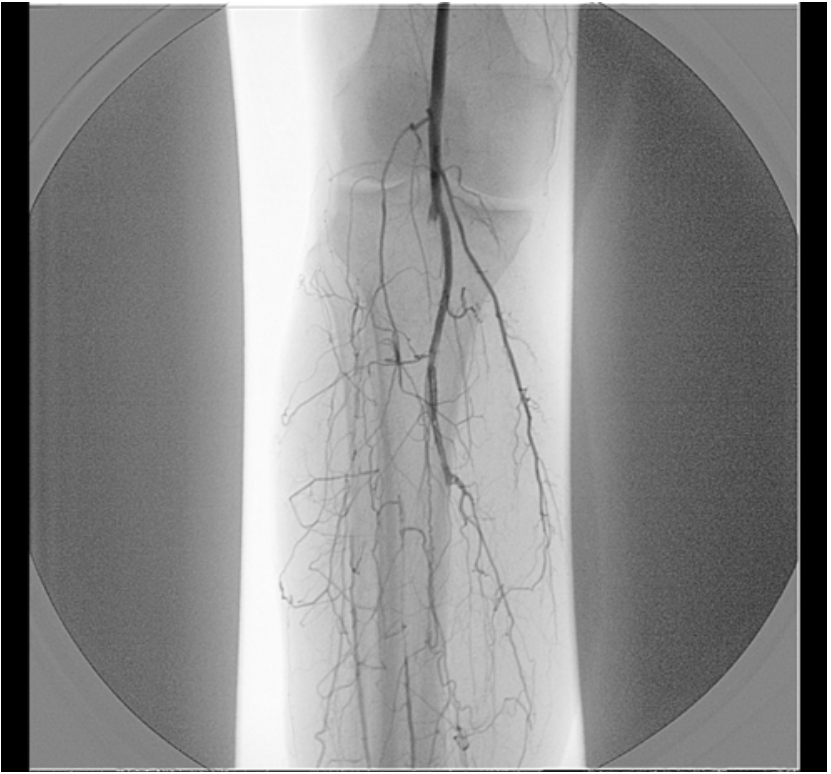

Figure 1 Digital subtraction angiography of the right leg in patient 1: occlusion of the popliteal artery at the level of the knee joint space and of the proximal crural vessels with thrombo-embolic appearance.

appearance. Percutaneous recanalization of the right popliteal artery was attempted by catheter-directed thrombolysis with urokinase followed by aspiration thrombectomy and percutaneous transluminal angioplasty (PTA). All procedures were unsuccessful. The patient was treated with oral anticoagulation and walking exercise. After 3 months the patient showed marked symptomatic and hemodynamic improvement.

\section{Case 2}

A 54-year-old, previously healthy white male presented at another hospital with acute, critical ischemia of the right leg. Angiography revealed occlusions of the right common and external iliac artery (Figure 2A) and of the left tibio-peroneal trunk. Surgical thrombectomy of the right iliac artery required two interventions because of early re-occlusion, and was unsuccessful in the left tibio-peroneal trunk. He underwent an extensive work-up including a 24-hour Holter monitor, transesophageal echocardiogram and aortic imaging to rule out embolic disease. At the time of discharge from the hospital ankle-brachial indices were normal and walking distance unlimited.

The patient was first seen at our clinic 8 months later complaining of a 5-month history of bilateral calf claudication. He had no history of diabetes, hypertension, hypercholesterolemia, and no family history of cardiovascular disease. He had quit smoking 1 year previously after a 12-pack year history.

Physical examination and non-invasive vascular laboratory tests showed bilateral PAD with impaired hemodynamic function at the level of the right thigh and left calf. Laboratory tests including markers of
(A)

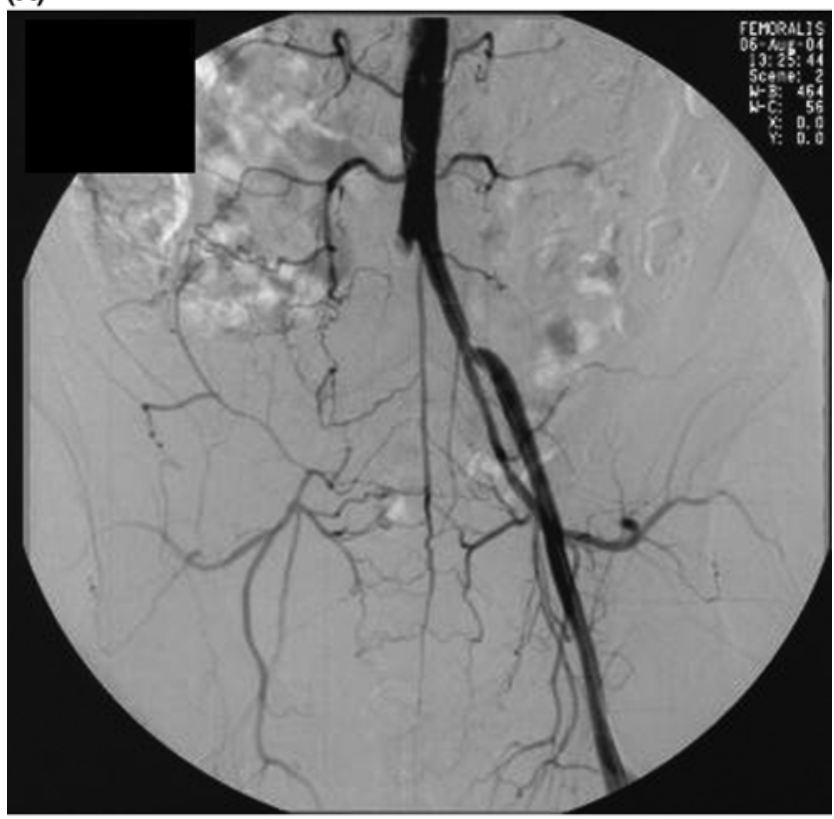

(B)

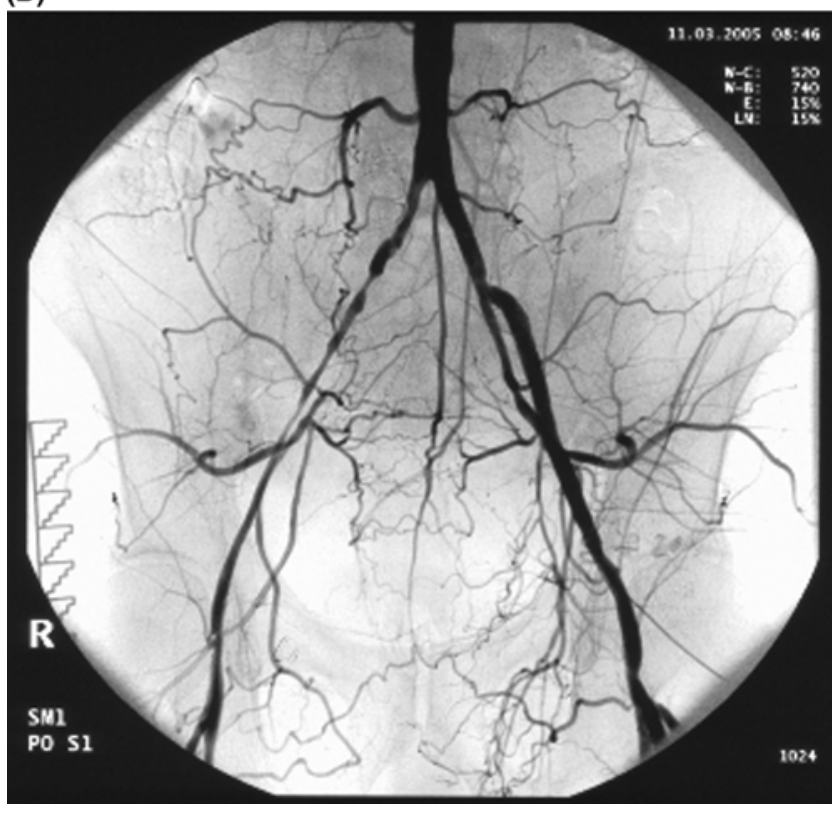

Figure 2 Digital subtraction angiography of the aortoiliac vessels in patient 2 at the time of initial presentation (A), and 8 months after surgical revascularization (B). The first angiogram shows acute, thrombo-embolic occlusion of the right iliac arteries, and the second angiogram shows high-grade, myointimal restenosis.

inflammation (ESR, CRP), electrophoresis, antinuclear and anticardiolipin antibodies and tests for thrombophilia (fibrinogen, ATIII, protein C and S, activated protein C) were normal. Homocysteine was $9.2 \mu \mathrm{mol} / 1$ (normal values $<13.9 \mu \mathrm{mol} / \mathrm{l}$ ). Lipid and lipoprotein analysis showed a borderline hypercholesterolemia (total cholesterol $209 \mathrm{mg} / \mathrm{dl}$, LDL-C $147 \mathrm{mg} / \mathrm{dl}$, HDL-C $40 \mathrm{mg} / \mathrm{dl}$, triglycerides $108 \mathrm{mg} / \mathrm{dl}$ ). $\mathrm{Lp}(\mathrm{a})$, measured with a commercial turbidometric 
immunoassay (Wako), was markedly elevated at $81 \mathrm{mg} / \mathrm{dl}$ (normal $>30 \mathrm{mg} / \mathrm{dl}$ ).

DSA performed at an outside facility a few days prior to presentation showed diffuse, high-grade restenosis of the right common and external iliac artery (Figure 2B), and of the left popliteal artery. Duplex ultrasound showed circular, hypoechogenic wall thickening in the affected arteries consistent with myointimal hyperplasia. We recommended PTA of the right iliac artery. The patient wished to undergo the intervention at an outside facility and was lost to follow-up. From a telephone contact, he is experiencing persistent bilateral claudication despite repeated interventions.

\section{Discussion}

We present the cases of two men with severe PAD due to multiple, thrombo-embolic arterial occlusions in the absence of advanced arteriosclerosis and traditional cardiovascular risk factors. Markedly elevated Lp(a) above $80 \mathrm{mg} / \mathrm{dl}$ was identified as the only significant laboratory abnormality. The morphology of the occlusions and the acute onset of symptoms suggest either embolism or local arterial thrombosis. A source of embolism could not be identified. However, embolism of unknown origin remains a possibility. Other etiologies of non-arteriosclerotic PAD such as thrombangiitis obliterans, large-vessel vasculitis, or popliteal entrapment have either been ruled out or appear very unlikely based on the clinical presentation and vascular morphology.

Elevated $\mathrm{Lp}(\mathrm{a})$ is a well-established risk factor for cardiovascular disease $\mathrm{e}^{2}$ and it has also been shown to be a strong predictor of peripheral vascular disease, independently of cigarette smoking and diabetes in a group of 100 white male patients with mean age of 67 years. ${ }^{5}$ In a case-control study of 50 white men under the age of 45 years with PAD, an elevated $\mathrm{Lp}$ (a) $(>30 \mathrm{mg} / \mathrm{dl})$ was an independent risk factor for the development of premature peripheral vascular disease. No association was found for homocysteine, antiphospholipid antibodies, antithrombin III, and protein $\mathrm{C}$ and $\mathrm{S}^{6}$ In a similar retrospective study in 55 white men, an elevated $L p(a)$ was specifically and independently associated with premature PAD but not with premature coronary artery disease. ${ }^{3}$ However, patients in these studies presumably had arteriosclerotic PAD. To our knowledge this is the first report of highly elevated $\mathrm{Lp}(\mathrm{a})$ in patients with an initial diagnosis of PAD due to acute arterial thrombo-embolic occlusions.

The structural homology of apolipoprotein (a) with plasminogen confers $\mathrm{Lp}(\mathrm{a})$ unique antifibrinolytic and prothrombotic properties. ${ }^{1,4}$ Several lines of evidence suggest that $L p(a)$ may be a risk factor for thrombotic vascular disorders unrelated to arteriosclerosis. Elevated $\mathrm{Lp}(\mathrm{a})$ has been linked to venous thrombo-embolism, ${ }^{7}$ childhood ischemic stroke, ${ }^{8}$ left atrial thrombus, ${ }^{9}$ and pregnancy loss. ${ }^{10}$

Another remarkable feature of the two presented cases is the unsatisfactory outcome after percutaneous and surgical vascular interventions. Elevated $\mathrm{Lp}(\mathrm{a})$ has been previously recognized as a risk factor for shortterm, myointimal restenosis after interventions in peripheral arteries. ${ }^{11}$ In a prospective study of 139 consecutive patients with successful PTA of the femoropopliteal area, the group who developed re-stenosis or re-occlusion within 1 year had a higher $\operatorname{Lp}(\mathrm{a})$ than the group without re-stenosis (59 vs $28 \mathrm{mg} / \mathrm{dl}$ ). This was independent of all other risk factors, which were equally distributed between the two groups. ${ }^{12}$ Moreover, Lp(a) has been shown to accumulate specifically at sites of initial myointimal hyperplasia in an animal model after arterial injury caused by balloon angioplasty. ${ }^{13}$

Patient 1 received testosterone replacement therapy prior to the occurrence of claudication. We cannot exclude that the hormonal therapy was a precipitating event for the thrombotic disease. The net effect of testosterone on the cardiovascular risk profile is unknown and both beneficial and deleterious effects have been recorded. ${ }^{14}$ We found one report of acute limb-threatening ischemia due to thrombosis of femoropopliteal and crural arteries after treatment with the synthetic androgen danazol, which was, however, mediated by severe thrombocytosis. ${ }^{15}$

Although our findings need to be confirmed in other cases, we believe that markedly elevated $\operatorname{Lp}$ (a) may have played an important causative role in the presented two cases of acute PAD due to thromboembolic arterial occlusions. We suggest that it may be worthwhile to measure $\mathrm{Lp}(\mathrm{a})$ in young patients with PAD with unexplained, acute, arterial occlusions and lack of other traditional risk factors.

\section{References}

1 Loscalzo J. Lipoprotein(a). A unique risk factor for atherothrombotic disease. Arteriosclerosis 1990; 10: 672-79.

2 Danesh J, Collins R, Peto R. Lipoprotein(a) and coronary heart disease: meta-analysis of prospective studies. Circulation 2000; 102: 1082-85.

3 Valentine RJ, Grayburn PA, Vega GL, Grundy SM. Lipoprotein (a) is an independent, discriminating risk factor for premature peripheral atherosclerosis among white men. Arch Intern Med 1994; 154: 801-806.

4 Koschinsky ML. Lipoprotein (a) and the link between atherosclerosis and thrombosis. Can J Cardiol 2004; 20 (suppl B): 37B-43B.

5 Widmann MD, Sumpio BE. Lipoprotein (a): a risk factor for peripheral vascular disease. Ann Vasc Surg 1993; 7: 446-51.

6 Valentine RJ, Kaplan KS, Green R, Jacobsen DW, Myers SI, Clagett GP. Lipoprotein (a), homocysteine, and hypercoagulable states in young men with premature peripheral atherosclerosis: a prospective, controlled analysis. J Vasc Surg 1996; 23: 53-61. 
7 Marcucci R, Liotta AA, Cellai AP et al. Increased plasma levels of lipoprotein (a) and the risk of idiopathic and recurrent venous thromboembolism. Am J Med 2003; 115: 667-68.

8 Novak-Gottl U, Strater R, Heinecke A et al. Lipoprotein(a) and genetic polymorphisms of clotting factor $\mathrm{V}$, prothrombin, and methylenetetrahydrofolate reductase are risk factors of spontaneous ischemic stroke in childhood. Blood 1999; 94: 3678-82.

9 Igarashi Y, Yamaura M, Ito M, Inuzuka H, Ojima K, Aizawa Y. Elevated serum lipoprotein(a) is a risk factor for left atrial thrombus in patients with chronic atrial fibrillation: a transesophageal echocardiographic study. Am Heart J 1998; 139 555-56.

10 Krause M, Sonntag B, Klamroth R et al. Lipoprotein(a) and other prothrombotic risk factors in Caucasian women with unexplained recurrent miscarriage. Results of a multicentre case-control study. Thromb Haemost 2005; 93: 867-71.
11 Lippi G, Veraldi GF, Dorucci V et al. Usefulness of lipids, lipoprotein(a) and fibrinogen measurements in identifying subjects at risk of occlusive complications following vascular and endovascular surgery. Scand J Clin Lab Invest 1998; 58: 497-504.

12 Maca T, Ahmadi R, Derfler K et al. Elevated lipoprotein (a) and increased incidence of restenosis after femoropopliteal PTA. Rationale for the higher risk of recurrence in females? Atherosclerosis 1996; 127: 27-34.

13 Ryan MJ, Emig LL, Hicks GW et al. Localization of lipoprotein(a) in a monkey model of rapid neointimal growth. Arterioscler Thromb Vasc Biol 1997; 17: 181-87.

$14 \mathrm{Wu}$ FC, von Eckardstein A. Androgens and coronary artery disease. Endocr Rev 2003; 24: 183-217.

15 Alvarado RG, Liu JY, Zwolak RM. Danazol and limbthreatening arterial thrombosis: two case reports. J Vasc Surg 2001; 34: 1123-26. 